Provided for non-commercial research and education use. Not for reproduction, distribution or commercial use.

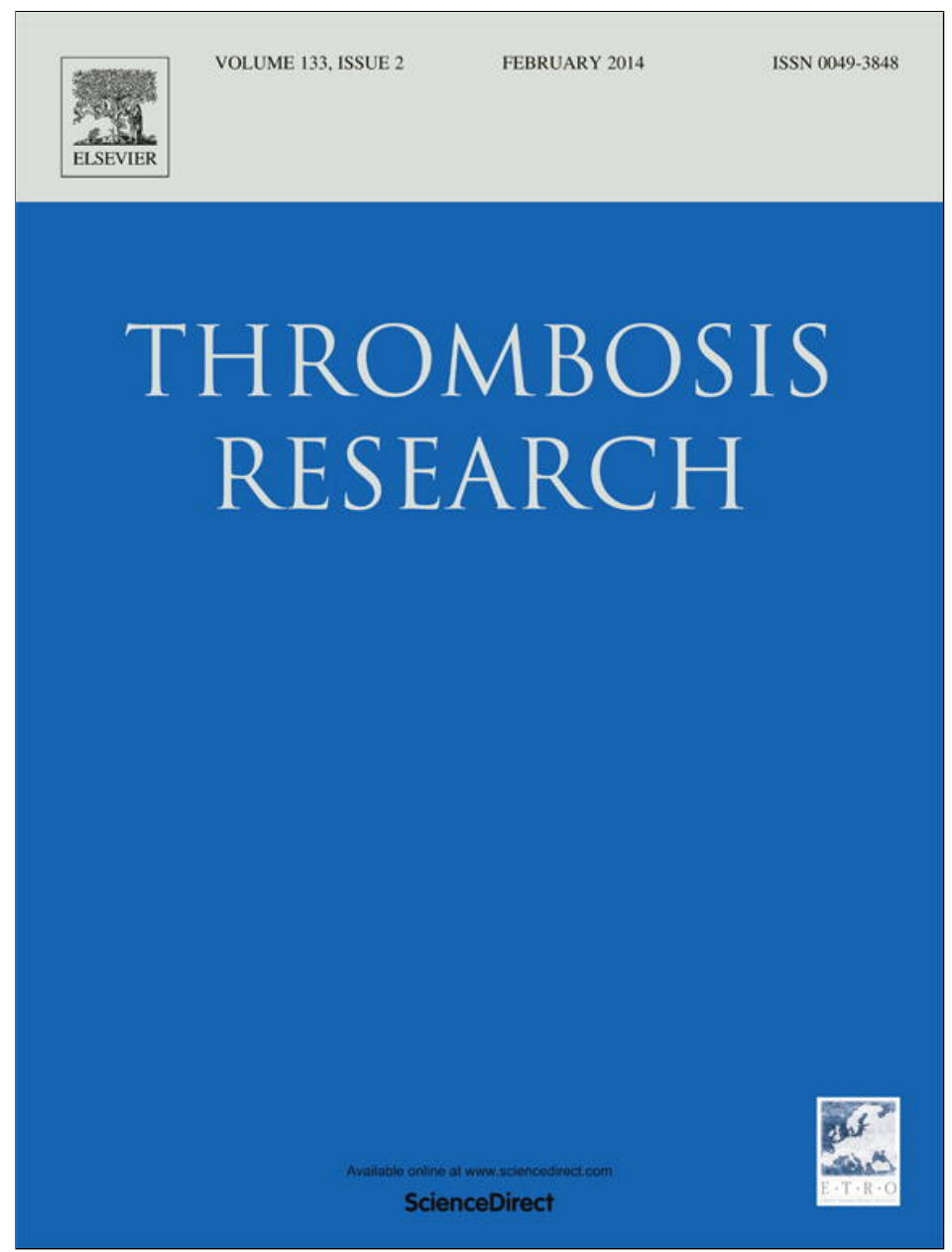

This article appeared in a journal published by Elsevier. The attached copy is furnished to the author for internal non-commercial research and education use, including for instruction at the authors institution and sharing with colleagues.

Other uses, including reproduction and distribution, or selling or licensing copies, or posting to personal, institutional or third party websites are prohibited.

In most cases authors are permitted to post their version of the article (e.g. in Word or Tex form) to their personal website or institutional repository. Authors requiring further information regarding Elsevier's archiving and manuscript policies are encouraged to visit:

http://www.elsevier.com/authorsrights 
Regular Article

\title{
Improved circulating microparticle analysis in acid-citrate dextrose (ACD) anticoagulant tube
}

\author{
Bence György ${ }^{\mathrm{a}, *}$, Krisztina Pálóczi ${ }^{\mathrm{a}}$, Alexandra Kovács ${ }^{\mathrm{a}}$, Eszter Barabás ${ }^{\mathrm{b}}$, Gabriella Bekő ${ }^{\mathrm{b}}$, Katalin Várnai ${ }^{\mathrm{b}}$, \\ Éva Pállinger ${ }^{\mathrm{a}}$, Katalin Szabó-Taylor ${ }^{\mathrm{a}}$, Tamás G. Szabó ${ }^{\mathrm{a}}$, Attila A. Kiss ${ }^{\mathrm{c}}$, András Falus ${ }^{\mathrm{a}}$, Edit I. Buzás a,** \\ a Semmelweis University, Department of Genetics, Cell- and Immunobiology, Budapest, Hungary \\ b Semmelweis University, Department of Laboratory Medicine, Budapest, Hungary \\ c Military Hospital, National Health Institute, Department of Obstetrics and Gynecology, Budapest, Hungary
}

\section{A R T I C L E I N F O}

\section{Article history:}

Received 15 July 2013

Received in revised form 13 September 2013

Accepted 18 November 2013

Available online 25 November 2013

\section{Keywords:}

ACD

Extracellular vesicle

Flow cytometry

Microparticles

Microvesicles

\begin{abstract}
A B S T R A C T
Introduction: Recently extracellular vesicles (exosomes, microparticles also referred to as microvesicles and apoptotic bodies) have attracted substantial interest as potential biomarkers and therapeutic vehicles. However analysis of microparticles in biological fluids is confounded by many factors such as the activation of cells in the blood collection tube that leads to in vitro vesiculation. In this study we aimed at identifying an anticoagulant that prevents in vitro vesiculation in blood plasma samples.

Materials and Methods: We compared the levels of platelet microparticles and non-platelet-derived microparticles in platelet-free plasma samples of healthy donors. Platelet-free plasma samples were isolated using different anticoagulant tubes, and were analyzed by flow cytometry and Zymuphen assay. The extent of in vitro vesiculation was compared in citrate and acid-citrate-dextrose (ACD) tubes.

Results: Agitation and storage of blood samples at $37{ }^{\circ} \mathrm{C}$ for 1 hour induced a strong release of both platelet microparticles and non-platelet-derived microparticles. Strikingly, in vitro vesiculation related to blood sample handling and storage was prevented in samples in ACD tubes. Importantly, microparticle levels elevated in vivo remained detectable in ACD tubes.

Conclusions: We propose the general use of the ACD tube instead of other conventional anticoagulant tubes for the assessment of plasma microparticles since it gives a more realistic picture of the in vivo levels of circulating microparticles and does not interfere with downstream protein or RNA analyses.
\end{abstract}

(C) 2013 Elsevier Ltd. All rights reserved.

\section{Introduction}

Extracellular vesicles (EVs) are membrane surrounded structures of various sizes (30-5000 $\mathrm{nm}$ ) that have received significant attention recently [1]. EVs may be classified on the basis of their biogenesis, diameter and membrane markers. The two best characterized types of EVs include exosomes of endosomal origin and plasma membrane-derived microparticles (MPs) (recently often referred to also as microvesicles or ectosomes in the literature) [1]. EVs are present in all biological fluids

Abbreviations: ACD, acid - citrate - dextrose; AX, annexin V; CTAD, citrate theophylline - adenosine - dipyridamole; CPDA, citrate- phosphate -dextrose adenin; EDTA, ethylenediaminetetraacetate; EV, extracellular vesicles; ISTH, International Society on Thrombosis and Haemostasis; FITC, fluorescein isothiocyanate; MP, microparticles; PerCP, peridinin chlorophyll protein; PCR, polymerase chain reaction; PFP, platelet free plasm; PMP, platelet microparticle; PS, phosphatidylserine; RMS, root mean square; RT, room temperature.

* Correspondence to: B. György, Nagyvárad tér 4, H-1089 Budapest, Hungary. Tel.: + 36 1 4591500/56432; fax: + 3613036968.

** Correspondence to: E.I. Buzás, Nagyvárad tér 4, H-1089 Budapest, Hungary. Tel.: + 36 12102929 ; fax: + 3613036968 .

E-mail addresses: gyorgyben@gmail.com (B. György), edit.buzas@gmail.com (E.I. Buzás). including blood plasma, synovial fluid, cerebrospinal fluid, urine, tears and breast milk [1]. MPs are in between 100 and $1000 \mathrm{~nm}$ in diameter, and they are also detectable by flow cytometry [2,3]. Thus, MP profiles are easily analyzed in the routine clinical laboratory practice, and represent novel biomarkers of various diseases. In circulation, most MPs are derived from platelets, red blood cells, endothelial cells and leukocytes. Because of their abundance, platelet-derived MPs (PMPs) received the highest attention during the past few years. Elevated PMP counts are characteristic for nearly all autoimmune disorders [4,5], and also for several cardiovascular and metabolic diseases [1]. EVs not only contain proteins but also RNA molecules [1]. Extracellular RNA (exRNA) in blood plasma is encapsulated in EVs, or bound to either proteins or HDL molecules [6]. exRNAs, particularly miRNAs, are specific and sensitive biomarkers of various diseases (for a review see [7]).

Although MPs represent promising novel biomarkers, their precise analysis is confounded by several pre-analytical factors (e.g. blood sampling, transportation and centrifugation of blood $[8,9]$ ) and analytical issues [10]. Well-known confounding factors include i) artificial, in vitro activation of platelets in the blood collection tube induced by agitation or transportation [3], ii) residual platelets present in „platelet-free plasma” [11], iii) detection threshold of the used 
flow cytometer [2] and iv) presence of protein aggregates [12] or calcium phosphate microprecipitates [13] that mimic MPs during flow cytometry. Recently, in joint efforts supervised by the International Society on Thrombosis and Haemostasis (ISTH), we and others suggested standard pre-analytical and analytical procedures for the detection of MPs $[2,3,10]$. This will facilitate the improved flow cytometry measurement of these structures. However, there are remaining debated questions including the use of anticoagulants for MP analysis. Although most studies used sodium-citrate tubes, some groups applied ACD (acid-citrate-dextrose) tubes in their studies [14-16]. A recent report by Jayachandran et al. suggested that neither citrate nor ACD tubes were suitable for MP measurement as these anticoagulants may eliminate MPs from plasma [17]. In contrast to the ISTH protocol, the above authors suggested the use of heparin as an anticoagulant. Furthermore, they proposed that published data on blood plasma MP levels using sodium citrate or ACD as an anticoagulant may need re-evaluation [17]. This debate prompted us to carry out a systemic analysis on the effect of anticoagulants on blood plasma MP levels. In this study we found that ACD stabilizes MP count in the blood collection tube by inhibiting in vitro vesiculation. Therefore we suggest the use of ACD tubes in order to obtain a realistic picture of in vivo MP levels without interfering with RNA analysis.

\section{Materials and Methods}

\section{Blood Donors}

We collected venous blood samples from healthy volunteers into blood collection tubes containing different anticoagulants. The donors did not take any medications in the last 3 months prior to blood sampling, nor did they suffer from any chronic or acute disease at the time of venipuncture. Smoking habit was documented. For the comparison of different anticoagulants, we tested 30 healthy individuals ( 14 females, 16 males, mean age \pm s.d.: $30.2 \pm 11.0$ years, range: 22-59 years, 4 smokers). For these individuals, a routine blood test was performed, and hemoglobin, platelet count, white blood cell count, blood sugar, C-reactive protein, creatinine, gamma-glutamyl transpeptidase, alanine aminotransferase and aspartate aminotransferase values were documented. These parameters were in the normal range of all included subjects. To test the effect of citrate and ACD tubes on artificially induced, in vitro vesiculation, we collected blood plasma from further 6 healthy individuals ( 3 females, 3 males, mean age \pm s.d.: $24.8 \pm 5.3$ years, age range: $18-34$ years, nonsmokers). To test the individual components of ACD (citric acid and dextrose) on the inhibition of artificially induced vesiculation, we also recruited 10 healthy individuals ( 7 women, 3 men, mean age \pm s.d.: 38.8 years \pm 10.5 years, age range: $23-53$ years, all non-smokers). Resistive pulse sensing experiments were carried out testing the blood plasma of 3 individuals ( 2 women, 1 man, mean age \pm s.d.: $33.0 \pm 15.9$ years). Furthermore, we collected blood plasma samples from non-smoker pregnant women (6 women, mean age \pm s.d.: $30.8 \pm 1.3$ years, range: $29-33$ years, all in the third trimester), and 6 age matched healthy, non-pregnant, non-smoker women as controls (mean age \pm s.d.: $27.8 \pm 5.2$ years, range: $23-37$ years). During the entire investigation period, we followed the guidelines and regulations of the Helsinki Declaration in 1975, and the experiments were approved by the Hungarian Scientific and Research Ethics Committee; all tested individuals signed an informed consent form.

\section{Blood Collection}

During the entire investigation, we rigorously followed the guidelines ISTH on blood sampling and handling for MP analysis $[3,9]$, except for the use of various blood collection tubes containing different anticoagulants. In the present study we used tubes containing citrate (sodium-citrate $3.2 \%, 3.5 \mathrm{ml}$ volume, art. no.: 454332, Greiner
Bio-One, Frickenhausen, Germany), heparin (lithium-heparin, $3 \mathrm{ml}$ volume, art no.: 454244, Greiner Bio-One), EDTA ( $\mathrm{K}_{3}$ EDTA, $3 \mathrm{ml}$ volume, art. no.: 454217), acid-citrate dextrose tube (ACD-A tube, $9 \mathrm{ml}$ volume, art. no.: 455055, Greiner Bio-One), citrate-theophyllineadenosine-dipyridamole tube (CTAD, $3.5 \mathrm{ml}$ volume, art. no.: 454462) and citrate phosphate dextrose adenine tube (CPDA, $6 \mathrm{ml}$ volume, art. no.: 456057). The venipuncture was performed in the morning (8-11 hours am), while the donors were fasting. We used a 21 Gauge needle for the venipuncture of an antecubital vein after applying a light tourniquet. The first few milliliters of blood were discarded, and were not used subsequently for MP analysis. The blood collection tubes were turned up and down five times gently in order to mix anticoagulants with blood. The venipuncture was performed within the same laboratory as blood sampling, thus, samples were not transported. The blood collection tubes were held in a rack in an upright position until centrifugation at room temperature (RT). During blood sampling and centrifugation the sequence of order of tubes with different anticoagulants was randomized. The centrifugation was performed within one hour after venipuncture. The different anticoagulant tubes were handled exactly the same manner by the same investigator.

\section{Preparation of Platelet-Free Plasma (PFP)}

We applied the ISTH protocol for preparation of PFP [3,9]. The blood was centrifuged at 2,500 $\mathrm{g}$ at room temperature using a Hermle Z206A table-top centrifuge (Hermle Labortechnik $\mathrm{GmbH}$, Wehingen, Germany). After 15 minutes of centrifugation, the platelet-poor plasma (PPP) was aspirated. At least $500 \mu \mathrm{L}$ of PPP was left in the tube in order to minimize contamination with cells. The PPP samples were then centrifuged once again at 2,500 g for 15 minutes. The PFP was then collected (again, at least $100 \mu \mathrm{L}$ was left in the polypropylene tubes), aliquoted, snap frozen in liquid nitrogen, and stored at $-80^{\circ} \mathrm{C}$ degrees until analysis. Snap freezing of the samples did not result in any significant alteration in the MP count measured by flow cytometry in the case of citrate and ACD tubes ( $n=9$, Supplementary Fig. 1 ).

\section{Flow Cytometric Analysis of PFP Samples}

PFP samples were analyzed using a FACSCalibur flow cytometer (BD Biosciences, Franklin Lakes, NJ, USA). The flow cytometry instrument settings and MP gating were adopted from previous works $[2,12,16]$. We used Megamix beads (BioCytex, Marseille, France) to determine the gate for MPs, as described previously [2,18]. Briefly, $0.5 \mu \mathrm{m}, 0.9 \mu \mathrm{m}$ and $3 \mu \mathrm{m}$ beads were detected on an SSC/FL2 plot (Supplementary Fig. 2A). Next, we selected bead regions, and backgated them on to the FSC/SSC plot (Supplementary Fig. 2B). MP gate was set on the FSC/SSC plot as described previously $[2,12,18]$ (Supplementary Fig. 2B and C). Counting beads ( $3 \mu \mathrm{m}$ in diameter, Partec GmbH, Münster, Germany) were detected on the SSC/FL2 plot (Supplementary Fig. 2D). We applied our earlier protocol for staining of MPs in biological fluids [12]. Briefly, $1 \mu \mathrm{g}$ of anti-CD42aPerCP antibody (BD Biosciences) was added to $20 \mu \mathrm{L}$ PFP, and incubated for 30 minutes at RT in dark. The samples were then diluted up to $400 \mu \mathrm{L}$ in $0.9 \%$ sodium-chloride. Next, we added $2 \mu \mathrm{L}$ of annexin V-FITC (AX-FITC, BD Biosciences) to the samples. The amounts of AXFITC and anti-CD42a antibodies were determined in preliminary tests. Before measurement, $\mathrm{Ca}^{2+}$ was added to the samples up to a concentration of $2.5 \mathrm{mM}$, and samples were incubated for 5 minutes. This calcium concentration resulted in maximal AX binding (as determined in preliminary measurements), in accordance with previous works [19], except for EDTA tubes. EDTA is a strong chelator of $\mathrm{Ca}^{2+}$, whose presence interferes with AX binding. Therefore we excluded EDTA samples from subsequent analysis in order to analyze all samples at the same $\mathrm{Ca}^{2+}$ concentration. Event numbers of equal sample volumes were counted for 60 seconds at medium flow rate. 
Background fluorescence was compared with that of the isotypecontrol antibody. When detecting AX binding, 5 mM EDTA-containing annexin-binding buffer solution was used to determine the background fluorescence. PMP $\left(\mathrm{CD} 42^{+} \mathrm{AX}^{+}\right)$and non-PMP $\left(\mathrm{CD} 42^{-} \mathrm{AX}{ }^{+}\right)$counts were enumerated (Supplementary Fig. $2 \mathrm{E}$ ). They were referred to known amounts of fluorescent counting beads detected on the FL2 plot (Supplementary Fig. 2D). MP concentration was calculated using the following formula: (MP concentration $)=(\mathrm{MP}$ count/bead count $)$ $\mathrm{x}$ bead concentration $\mathrm{x}$ plasma dilution. To verify if we detected MPs (vesicular structures), and to exclude the presence of immune complexes or protein aggregates, we added $0.1 \%$ Triton X-100 to the samples, as we described previously [5,12]. This step resulted in prompt disappearance of fluorescent event counts (Supplementary Fig. 2F) suggesting the presence of membranous structures within the MP gate. All measurements were carried out in $\mathrm{NaCl}$ solution instead of PBS in order to avoid calcium microprecipitation [13].

\section{Resistive Pulse Sensing}

To test the size distributions and concentrations of MPs in blood plasma anticoagulated with citrate or ACD, we performed resistive pulse sensing analysis using a qNano instrument (Izon Science Ltd., Christchurch, New Zealand) [20]. The use of the $200 \mathrm{~nm}$ nanopore membrane enabled us to cover the typical MP size range in the blood plasma [12,21]. PFP samples were diluted $1: 1$ in $0.1 \mu \mathrm{m}$ filtered PBS. This dilution was selected by previous serial dilution experiments testing samples from undiluted ones to 1:1000 dilution. MPs were counted for 5 minutes using 7.15 mbar pressure. Voltage was set in between 0.1-0.25 Volts in order to achieve a stable $100 \mathrm{nA}$ current. Particle histograms were recorded when RMS noise was below $12 \mathrm{pA}$, and particle rate in time was linear.

\section{Prothrombinase Assay}

PFP samples were also analyzed by Zymuphen MP-Activity Kit (Hyphen, BioMed, Neuville-sur-Oise, France), a functional assay of the procoagulant activity of MPs. Briefly, 1:20 diluted PFP samples were applied onto plates coated with streptavidine and biotinylated AX-V. After incubation at $37{ }^{\circ} \mathrm{C}$ for one hour, plates were washed 5 times with wash buffer provided by the manufacturer. Next, bovine factor Xa-Va mixture and human prothrombin were added, and plates were incubated for 10 minutes at $37{ }^{\circ} \mathrm{C}$. After the addition of the thrombin-specific chromogenic substrate, the reaction was stopped with $2 \%$ citric acid, and absorbance was measured at $405 \mathrm{~nm}$. Calibrators were used, and values were expressed in phosphatidylserine (PS) equivalents (nM PS).

\section{Artificial Induction of In Vitro Vesiculation}

We tested artificially induced release of MPs from platelets and other cells in whole blood. We applied gentle, high frequency $(50 \mathrm{~Hz}$ ), low amplitude shaking (similar to motor-vehicle transportation) using a plate shaker (Denley WeWarm1, Cat No.: WI-031, DJB Labcare Ltd., Newport Pagnell, UK) for 1 hour at RT. We also tested the effects of 1 hour incubation both at $37{ }^{\circ} \mathrm{C}$ and RT. As a control, we measured PMP and non-PMP levels in PFP samples prepared immediately after blood sampling from the same donors.

\section{Calibrated Automated Thrombogram}

To analyze platelet function in citrate and ACD tubes, we used a calibrated automated thrombogram (CAT) assay. Platelet rich plasma (PRP) was prepared from blood plasma of 4 healthy individuals ( 3 females, 1 male, mean age \pm s.d.: $45.5 \pm 11.7$ years) using $180 \mathrm{~g}$ centrifugation for 6 minutes. Platelet count was determined and platelet concentration was set to $150,000 / \mu l$ by diluting the samples with
PFP. Reaction was started by mixing $80 \mu \mathrm{PRP}$ with $20 \mu$ activator (containing 1 pM tissue factor, Diagnostica Stago SAS, Nanterre, France) and $20 \mu \mathrm{l}$ fluorescent substrate (Diagnostica Stago), according to the manufacturer. Fluorescence was detected for 60 minutes using a Fluoroskan Ascent CAT machine (Thermo Scientific, Waltham, MA, USA).

\section{RNA Isolation and miRNA Analysis}

To analyze miRNA content of plasma and blood plasma derived EVs, we collected blood plasma samples into citrate and ACD tubes from 5 healthy donors ( 3 females, 2 males, mean age \pm s.d.: $28 \pm 3.7$ years). Total RNA was isolated from $150 \mu$ of PFP (filtered through a $0.8 \mu \mathrm{m}$ filter) using Qiagen miRNeasy Mini Kit (Qiagen, Germantown, MD, USA). We also isolated RNA from EVs isolated from the same PFP samples ( $8 \mathrm{~mL}$ volume, these vesicle samples contained both MPs and exosomes in the $100,000 \mathrm{~g}$ for 60 minutes pellets) using Qiagen RNeasy Plus Micro Kit (Qiagen). RNA quality was tested using RNA 6000 Pico Kits (Agilent, Santa Clara, CA, USA) and small RNA fractions were determined using a small RNA kit (Agilent) on an Agilent 2100 Bioanalyzer. In order to detect miRNAs both in PFP samples and EVs, we performed real-time PCR assays for miR16, miR24, miR451 and let7a. Reverse transcription of RNA was carried out using miRNA-specific stem-loop primers (hsa-miR-16 [RT 391]; hsa-miR-24 [RT 402]; hsa-miR-451 [RT 1105]; hsa-let-7a [RT 377], Life Technologies, Carlsbad, CA, USA) and TaqMan MicroRNA Reverse Transcription Kit (Life Technologies) on an Applied Biosystems GeneAmp® PCR System 9700 machine. qPCR was performed using SensiFAST ${ }^{\mathrm{TM}}$ Probe Hi-ROX One-Step Kit (Bioline, Taunton, MA, USA) and miRNA-specific primers and probes (Life Technologes) on an Applied Biosystems 7900HT Fast Real-Time PCR machine. cDNA product was visualized by gel electrophoresis in $10 \%$ polyacrylamide gels. We used a GeneRuler TM Ultra Low Range DNA Ladder $(0,5 \mu \mathrm{g} /$ lane)(Thermo Scientific). For analysis, we used a FluorChemTM 8000 Advanced Fluorescence machine (Alpha Innotech Corporation, San Jose, CA, USA).

\section{Statistical Analysis}

We compared multiple groups using Kruskal-Wallis one way analysis of variance on Ranks. Pairwise comparisons were carried out using Signed Rank test. To compare two non-related sample groups, we used Mann-Whitney Rank Sum test. For correlations, we used Pearson product-moment correlation. For statistical testing, we used the SigmaPlot for Windows, version 11.0 (Systat Software, Inc., San Jose CA, USA).

\section{Results}

Comparison of MP Counts in Different Anticoagulant Blood Collection Tubes

First we enumerated PMPs (CD42 $\left.{ }^{+} \mathrm{AX}^{+} \mathrm{MPs}\right)$ and non-PMPs $\left(\mathrm{CD} 42^{-} \mathrm{AX}^{+} \mathrm{MPs}\right)$ by flow cytometry in PFP samples collected into tubes with different anticoagulants (Fig. $1 \mathrm{~A}$ and $\mathrm{B}$ ). The PMP concentration in the citrate plasma samples was around $10^{2}-10^{4} / \mu \mathrm{l}$ in our study (median (1st quartile to 3rd quartile): $1,152 / \mu \mathrm{l}(251 / \mu \mathrm{l}-4,553 / \mu \mathrm{l})$ ), consistent with previous findings [10]. The type of anticoagulant significantly influenced both the PMP and non-PMP levels ( $p=0.002$ for PMPs and $\mathrm{p}<0.001$ for non-PMPs, Kruskal-Wallis one way analysis of variance on Ranks). The highest PMP and non-PMP counts were found in heparin tubes, and differed significantly from PMP and non-PMP counts in citrate tubes ( $p=0.012$ and $p<0.001$, respectively, Signed Rank test). The citrate, CTAD and CPDA tubes contained nearly equal amounts of MPs. The lowest MP count was detected in ACD tubes. The PMP count in this tube differed significantly from that of citrate tube ( $p=0.04$, Signed Rank test). Furthermore, inter-individual differences (reflected by standard deviation) of healthy samples were also the lowest in the case of the ACD tube samples. Using the 

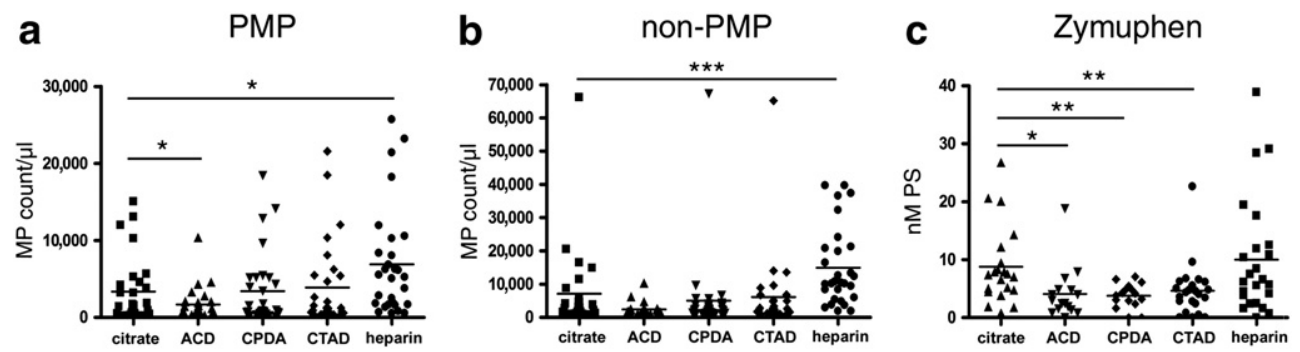

Fig. 1. Comparison of the effect of different anticoagulants on the MP counts measured by flow cytometry and Zymuphen assay. $P M P\left(C D 42 a^{+} A X^{+}\right)(a)$ and non-PMP $\left(C D 42 a^{-} A X^{+}\right)$ (b) counts enumerated by flow cytometry, (c) results of Zymuphen assay. Horizontal lines represent mean values. ${ }^{*} \mathrm{p}<0.05,{ }^{* *} \mathrm{p}<0.01,{ }^{* * *} \mathrm{p}<0.001, \mathrm{n}=30$.

Zymuphen assay, we obtained similar results (Fig. 1C). The median (1st quartile to 3rd quartile) PS concentration was $7.4 \mathrm{nM}(4.7-$ $10.1 \mathrm{nM})$ in the citrate samples, which was consistent with the healthy range determined by the manufacturer. Similarly to flow cytometry results, the plasma samples in heparin tubes showed the highest PS concentration (however, the difference was not significant compared to citrate tubes). On the contrary, ACD, CTAD, CPDA tubes contained significantly less PS as compared to citrate tubes ( $p=0.017, p=0.003$ and $p=0.006$, respectively, Signed Rank test)

Next we assessed if there was a correlation between flow cytometry and Zymuphen assay results (Supplementary Fig. 3). Zymuphen assay detects all PS positive structures including small MPs and exosomes invisible for many flow cytometers. We analyzed the correlation between all $\mathrm{AX}^{+} \mathrm{MPs}$ detected by flow cytometry (the sum of PMPs and nonPMPs) and Zymuphen assay results. There was only a tendency between the two methods in the case of citrate tubes $(\mathrm{R}=0.385$, Pearson correlation), and there was no correlation in the case of CPDA, CTAD and heparin tubes. Of note, there was a correlation in the case of ACD tubes $(\mathrm{R}=0.794, \mathrm{p}=0.00005$, Pearson correlation).

The lowest MP numbers and lowest standard deviation detected in the ACD tubes might indicate that artificial, in vitro vesiculation is limited in ACD tubes, compared to other tubes. Based on these findings we selected ACD tube as a candidate optimal anticoagulant, and in the rest of this study, we compared artificial in vitro vesiculation in ACD tube and citrate tubes (the latter being suggested by the latest ISTH recommendation [3]).

\section{Artificially Induced Vesiculation in Citrate and ACD Tubes}

We induced in vitro release of MPs in whole blood by gentle agitation and incubation of the blood samples at various temperatures. In accordance with previous reports [9], agitation of whole blood in citrated tubes resulted in a dramatic, however, highly variable increase (3-29 fold) of both PMP and non-PMP counts compared to untreated controls (Fig. 2A and B) $(p=0.03$ and $p=0.03$, respectively, Signed Rank test), measured by flow cytometry.

Similarly, incubation for one hour at $37{ }^{\circ} \mathrm{C}$ resulted in $2-3$ fold elevation of PMP and non-PMP counts in citrate tubes $(\mathrm{p}=0.03$ and $p=0.03$, respectively, Signed Rank test). Strikingly, in ACD tubes, handled exactly the same manner as citrate tubes, there was only moderate, non significant elevation of PMPs and non-PMPs both after gentle agitation and $37^{\circ} \mathrm{C}$ incubation (Fig. $2 \mathrm{~A}$ and B). The incubation of whole blood at RT for one hour did not result in significant changes in MP levels either in the case of citrate or ACD tubes. Using the Zymuphen assay, we observed similar results: in plasma samples from citrate tubes, PS concentration was elevated after agitation and $37{ }^{\circ} \mathrm{C}$ incubation $(\mathrm{n}=3$ ) (Fig. 2C). Similarly, one hour incubation at RT also resulted in an average of 5-fold elevation in PFP samples in citrate tubes. Most importantly, in ACD tubes, PS concentration remained unchanged after both agitation and incubation either at $37{ }^{\circ} \mathrm{C}$ or RT (Fig. 2C).

These data strongly support that in ACD tubes, artificially induced (in vitro) vesiculation of blood cells is successfully prevented.

\section{The Effect of Citric Acid and Dextrose on Artificially Induced Vesiculation}

To investigate which component of the ACD tubes was responsible for the inhibition of in vitro vesiculation, we analyzed the effect of citric acid and dextrose. These substances were added separately to the blood, drawn into conventional citrate tubes up to a concentration characteristic for the commercial ACD tube ( $8 \mathrm{~g} / \mathrm{L}$ citric acid and $24.5 \mathrm{~g} / \mathrm{L}$ dextrose). Then, the tubes were subjected to gentle agitation or $37{ }^{\circ} \mathrm{C} 1$ hour incubation, as described above. The addition of citrate successfully inhibited the release of PMPs during both agitation and $37^{\circ} \mathrm{C}$ incubation (Signed Rank test, $\mathrm{p}=0.01$ and $\mathrm{p}=0.016$, respectively) (Fig. 3A). The formation of non-PMP counts was also inhibited by citric acid during $37^{\circ} \mathrm{C}$ incubation ( $p=0.016$ ), but the release of PMPs was not inhibited during shaking (Fig. 3B). Dextrose alone could not inhibit the formation of either PMPs or non-PMPs counts during any of the tested conditions (Fig. $3 \mathrm{~A}$ and $\mathrm{B}$ ).

These data suggest that the active component of ACD tubes that inhibits artificial in vitro vesiculation, is citric acid which decreases the $\mathrm{pH}$ of the blood sample to approximately 6.0 [22].

To confirm that platelets are inhibited in ACD tubes (that do not contain direct platelet inhibitors), we performed CAT measurement of PRP samples in citrate or ACD tubes. Clearly, there was a significant delay in the activation of platelets, the velocity of activation was smaller and the
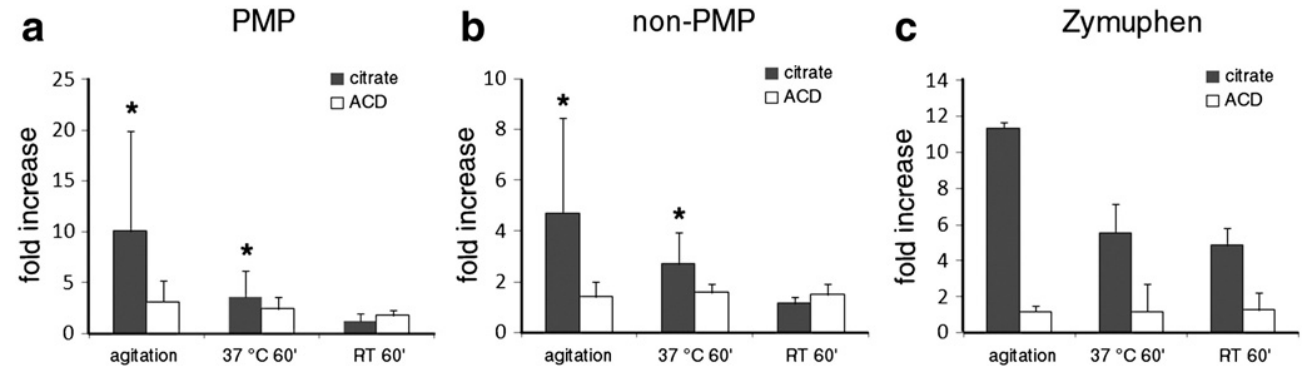

Fig. 2. The effect of citrate or ACD on the in vitro release of MPs. Counts of PMPs (a) and non-PMPs (b) measured by flow cytometry, and Zymuphen assay (c). The $y$ axis represents fold increase compared to PFP samples isolated immediately after blood sampling. RT denotes room temperature. Mean \pm s.d. values are shown. *p $<0.05$ compared to untreated control samples (Signed Rank test), $\mathrm{n}=6$. 

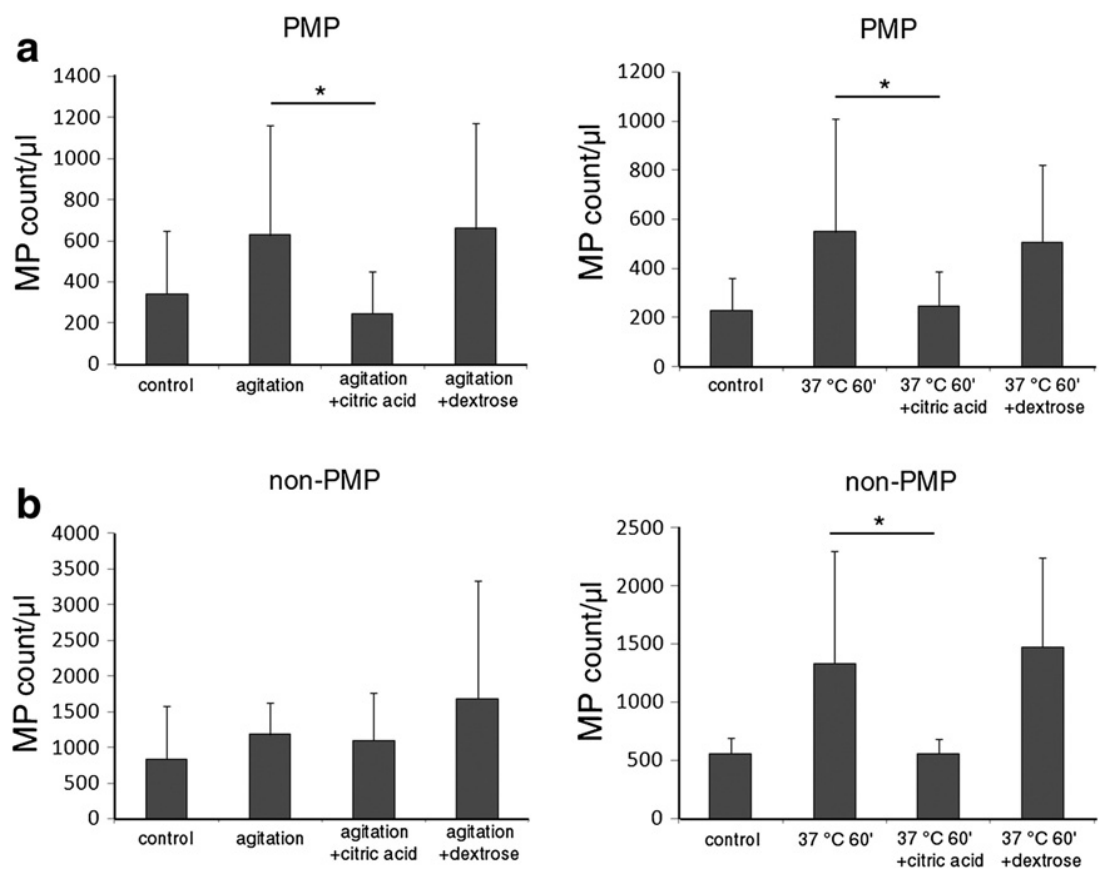

Fig. 3. The effect of ACD components on the in vitro release of PMPs (a) and non-PMPs (b) determined by flow cytometry. Citric acid or dextrose was added separately to the tubes, and blood samples were subsequently subjected to agitation or 1 hour incubation at $37^{\circ} \mathrm{C}$. Mean \pm s.d. values are shown. ${ }^{*} \mathrm{p}<0.05, \mathrm{n}=10$.

amount of total thrombin activity was also impaired in tubes with ACD (Supplementary Fig. 4). These results indicate that platelet inhibition explains the fewer numbers of PMPs in the PFP samples from ACD tubes.

Detection of Pregnancy Related Elevation of PMP and non-PMP Counts is not Confounded in ACD Tubes

Next, we assessed if the MP count elevated in vivo was also detectable in ACD tubes in pregnancy (a condition where elevated levels of PMPs and non-PMPs were reported previously in citrate tubes [23]). Indeed, there was a significant elevation in the PMP and non-PMP counts in pregnant women compared to non-pregnant healthy controls in citrate tubes $(\mathrm{p}=0.002$ and $\mathrm{p}=0.004$, respectively, Mann-Whitney Rank Sum test) (Fig. 4). Importantly, the pregnancy-related elevation was also detectable in ACD tubes ( $p=0.015$ for PMPs and $p=0.002$ for non-PMPs, Mann-Whitney Rank Sum test) (Fig. 4).

\section{Size Distribution of MPs in Citrate and ACD Tubes}

Using the qNano instrument, we determined the size distributions of blood plasma MPs in both citrate and ACD tubes (Fig. 5).
We did not detect any significant difference between the vesicle sizes in the two types of anticoagulant tubes. The mean $( \pm$ s.d.) diameter of particles was $242.3 \mathrm{~nm}( \pm 5.8) \mathrm{nm}$ in the case of citrate tubes and $230.8( \pm 9.2) \mathrm{nm}$ in the case of ACD tubes. Concentration of particles was lower in ACD tubes (Fig. 5) as compared to those in citrate tubes (in accordance with data obtained by flow cytometry and Zymuphen assay).

\section{RNA Analysis of Blood Plasma and EVs from Citrate and ACD Tubes}

Based on the above data, we propose the use of ACD tubes for the assessment of circulating MPs. Recently, several studies analyzed exRNA content of vesicles or blood plasma (or serum) $[24,25]$. We tested ACD tubes for downstream RNA analysis. Blood plasma and total EV (including both exosomes and MPs in the pellets) RNA profiles were similar between conventional citrate and ACD tubes (Supplementary Fig. 5A). We detected almost exclusively small RNA molecules in all samples, $18 \mathrm{~S}$ and $28 \mathrm{~S}$ RNAs were absent. We next applied small RNA analysis to determine the percentage of miRNAs in EV samples. We found that $46.8 \pm 0.8 \%(175.1 \pm 51.9 \mathrm{pg} / \mu \mathrm{L})$ and $45.2 \pm 2.3 \%(164.3 \pm 77.1 \mathrm{pg} / \mu \mathrm{L})$ of small RNAs were miRNAs

\section{PMP}

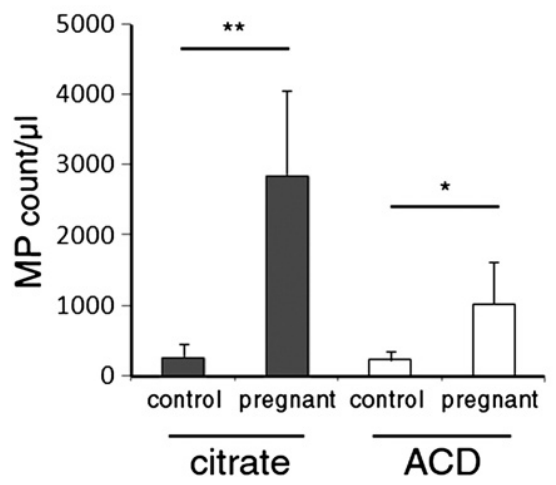

non-PMP

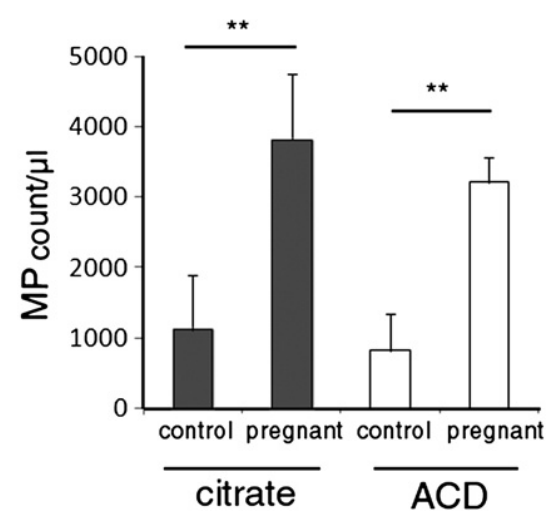

Fig. 4. MP counts in pregnancy measured in citrate and ACD tubes determined by flow cytometry. Mean \pm s.d. values are shown. ${ }^{*} \mathrm{p}<0.05,{ }^{* *} \mathrm{p}<0.01, \mathrm{n}=6$ in each group. 


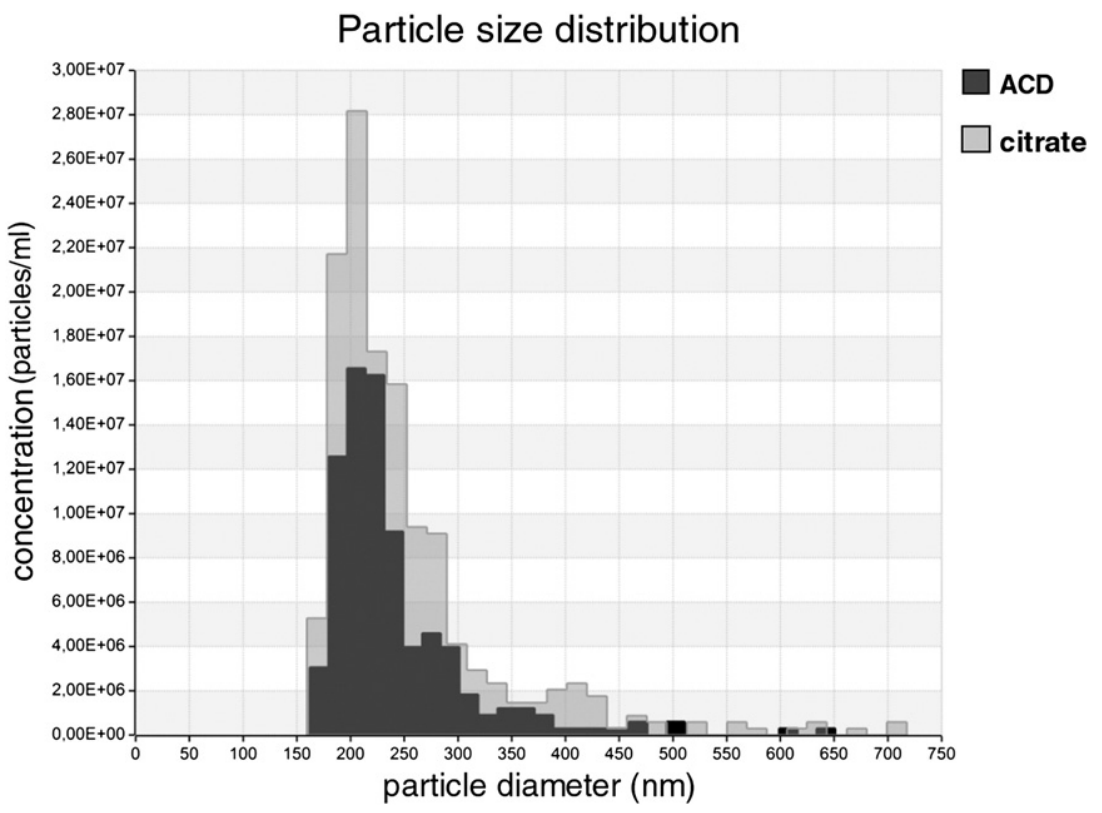

Fig. 5. Particle size distributions measured by resistive pulse sensing (qNano). Histograms show a representative PFP sample (diluted $1: 1$ in PBS), $\mathrm{n}=3$.

in citrate and ACD tubes, respectively. To further confirm the presence of miRNAs in the samples, we performed qRT-PCR analysis specific for miR16, miR24, miR451 and let7a. The presence of these miRNAs in blood or in vesicles has been confirmed earlier [24,25]. We found that all analyzed miRNAs were detectable in EVs and in blood plasma, both in the case of citrate and ACD tubes (Supplementary Fig. 5B). There was no significant difference in CT values for the above analyzed miRNAs between ACD and citrate tubes (equal amount of plasma was used for vesicle isolation or for RNA isolation).

\section{Discussion}

In this systemic study, we assessed whether the use of various anticoagulants affected the measured MP counts and in vitro vesiculation of blood cells. Indeed, we found that MP levels, determined by flow cytometry and Zymuphen assay, were significantly different in tubes with various anticoagulants. In accordance with previous results, the highest MP count was detected in tubes with heparin, and the lowest was found in ACD tubes [17]. This may be due to the fact that unlike other anticoagulants used in this study, heparin is not a calcium chelator, and calcium is known to play a crucial role in vesiculation $[26,27]$. We demonstrated that the use of citrate, a weak chelator of calcium, resulted in a significantly reduced number of MPs compared to heparin in the blood collection tube. Interestingly, we found comparable levels of PMP and non-PMP counts. This is in contrast with previous findings [1], as most previous works found PMPs the main MP population in blood plasma. However, many of these earlier works did not control for the presence of residual platelets, and did not use pre-analytical parameters suggested by the ISTH [3]. Thus, many of these vesicles might have corresponded to in vitro formed MPs. Recent standard protocols [3] use very efficient centrifugation and rigorous pre-analytical settings in order to remove most of platelets, resulting in lower number of PMPs.

In Zymuphen assay, tubes containing platelet inhibitors (CTAD, CPDA and ACD) showed decreased MP counts. In this assay, citrate tubes gave comparable results to heparin in contrast to data obtained by flow cytometry. This controversy may be explained by the fact that Zymuphen assay does not only detect all types of $\mathrm{AX}^{+} \mathrm{EVs}$ (even exosomes), but also any PS positive cells that may possibly remain in the PFP samples [11]. In contrast, during flow cytometry we gated on the MV size range only, and smaller or larger particles were thus, excluded from detection. By using the resistive pulse sensing approach, we detected much higher number of events than by flow cytometry. This is in accordance with previous reports [20], and is explained by the fact that flow cytometry is able to detect events above $200-300 \mathrm{~nm}$, while the threshold for qNano detection is lower. Furthermore, van der Pol et al. reported the presence of multiple vesicles in the laser beam (swarm effect) that was responsible for an up to 1000 -fold underestimation of MPs by flow cytometry [20].

Next, we focused on ACD and citrate tubes. Apparently, every single step from blood sampling to analysis may affect MP counts [8]. It has been shown previously that intense agitation of the tubes results in a strong elevation in MP count, and a significant decrease in the clotting time [9]. Furthermore, unsupported transportation and a delay between blood collection and the first centrifugation of samples also resulted in a significant increase in MP counts [9]. In the present study, we applied a high frequency and low amplitude gentle agitation, which induced an increase of PMP counts in citrate but not in ACD tubes. We also assessed the effect of blood sample storage at $37^{\circ} \mathrm{C}$ which increased both PMP and non-PMP counts in citrate tubes but not in ACD ones. Our results indicate that in vitro platelet vesiculation is prevented in ACD tubes compared to the commonly used citrate tubes. Most interestingly, tubes containing direct platelet inhibitors, like theophylline, adenosine and dipyridamole (CTAD tubes) could not inhibit in vitro vesiculation. ACD tubes contain dextrose and citric acid, which components were separately analyzed in our work. Dextrose had no effect on the counts of vesicles. Both in the case of PMPs and non-PMPs, citric acid was found responsible for the inhibition of vesiculation. The ACD tube was reported to result in $\mathrm{pH} 6.0$ value of the blood sample [22]. The authors reported that platelets prepared in ACD tubes, showed significantly fewer signs of activation compared to platelets in sodium-citrate tubes. Platelets retained their resting discoid morphology in ACD tubes, and the expression of the activation marker GPIIb-IIIa was also inhibited [22].

The inhibitory activity of local acidosis on platelets has been described long time ago [28]. Low pH was shown to inhibit ADPinduced change in platelet shape, adhesion, spreading and interaction with fibrinogen, fibronectin or collagen [29]. The mechanism is likely due to inhibition of the store-operated calcium influx into platelets in extracellular acidosis [30]. Lower intracellular calcium concentration results in the inhibition of haemostatic functions of platelets. As calcium 
is known to be required for vesiculation [26,27], it is not surprising that in vitro vesiculation was successfully dampened in the acidic environment. In this study, using CAT we also confirmed that platelets are markedly inhibited in ACD tubes. This inhibition might explain why PMP levels were generally lower in these tubes and why further effects related to blood handling failed to induce in vitro vesicle formation from platelets. However, a direct link between extracellular acidosis and vesiculation is yet to be established, and the inhibition of blood cells other than platelets in ACD tubes is yet to be confirmed.

Taken together, ACD tubes appear more suitable for MP analysis than any other types of anticoagulant tubes as they inhibit in vitro vesiculation. Importantly, detection of in vivo elevated MP counts was not prevented in ACD tubes.

Based on our data, if sufficient care is taken to handle blood tubes, if samples are centrifuged shortly after blood sample collection and benchtop storage temperature is at around RT, both citrate and ACD tubes give comparable results.

In the past couple of years, hundreds of articles assessed MPs from blood plasma using flow cytometry. The data, particularly on PMP counts, are often controversial; some studies show elevated levels of PMPs in a given condition, while some others fail to show an association (e.g. venous thromboembolism, [31]) One possible explanation for these results is the in vitro vesiculation of blood cells induced by many physical factors.

RNA profiles were found similar in citrate and ACD tubes. Mostly small RNAs were detected, and detailed Bioanalyzer analysis revealed that small RNA fractions contained miRNA in high amounts. The presence of miR16, miR24, miR451 and let7a in plasma has already been described [24,25], and we found no difference in the quantity of these RNAs in citrate and ACD tubes. Thus, as there was no difference in citrate and ACD tubes in terms of exRNA profiles and quantities of the studied specific miRNAs, ACD tubes are not only suitable for protein analysis of MVs, but also for exRNA extraction and subsequent analysis.

Taken together, we suggest the use of ACD tubes for clinical laboratory assessments of MP counts because in practice a strict control of transportation, temperature and delay between sample collection and analysis, is not always feasible. Conclusively, sample collection in ACD tubes may represent an important step towards standardization of both fresh and biobanked plasma samples for MP studies.

Supplementary data to this article can be found online at http://dx. doi.org/10.1016/j.thromres.2013.11.010.

\section{Conflict of Interest Statement}

The authors declare no conflicting interests.

\section{Acknowledgements}

This work was supported by OTKA K 73247, NK 84043 and K77537, Kerpel-Fronius Ödön Fellowship, Baross Gábor (REG-KM-09-1-20090010) and FP7-PEOPLE-2011-ITN - PITN-GA-2011-289033 "DYNANO" and BM1202 European Network on Microvesicles and Exosomes in Health and Disease (ME-HAD). Bence György and Tamás G Szabó are Kerpel-Fronius Ödön Fellows. This research was realized in the frames of TÁMOP 4.2.4. A/1-11-1-2012-0001 „National Excellence Program Elaborating and operating an inland student and researcher personal support system". The project was subsidized by the European Union and co-financed by the European Social Fund.

\section{Disclosure}

None declared.

\section{References}

[1] György B, Szabó TG, Pásztói M, Pál Z, Misják P, Aradi B, et al. Membrane vesicles, current state-of-the-art: emerging role of extracellular vesicles. Cell Mol Life Sci 2011;68:2667-88.

[2] Lacroix R, Robert S, Poncelet P, Kasthuri RS, Key NS. Dignat-George F; ISTH SSC Workshop. Standardization of platelet-derived microparticle enumeration by flow cytometry using calibrated beads: results of ISTH SSC collaborative workshop. J Thromb Haemost 2010;8:2571-4.

[3] Lacroix R, Judicone C, Mooberry M, Boucekine M, Key NS, Dignat-George F. The ISTH SSC Workshop. Standardization of pre-analytical variables in plasma microparticle determination: results of the International Society on Thrombosis and Haemostasis SSC Collaborative workshop. J Thromb Haemost Apr 2013. http://dx.doi.org/ 10.1111/jth.12207 [print copy in press. Available from: URL: http://onlinelibrary. wiley.com/doi/10.1111/jth.12207/abstract;jsessionid=A9B21C784EABD90B7B1556 CAFCBE8027.d02t02] [Epub ahead of print]PMID: 23551930 [PubMed - as supplied by publisher].

[4] Beyer C, Pisetsky DS. The role of microparticles in the pathogenesis of rheumatic diseases. Nat Rev Rheumatol 2010;6:21-9.

[5] György B, Szabó TG, Turiák L, Wright M, Herczeg P, Lédeczi Z, et al. Improved flow cytometric assessment reveals distinct microvesicle (cell-derived microparticle) signatures in joint diseases. PLoS One 2012;7:e49726.

[6] Vickers KC, Palmisano BT, Shoucri BM, Shamburek RD, Remaley AT. MicroRNAs are transported in plasma and delivered to recipient cells by high-density lipoproteins. Nat Cell Biol 2011;3:423-33.

[7] Weiland M, Gao XH, Zhou L, Mi QS. Small RNAs have a large impact: circulating microRNAs as biomarkers for human diseases. RNA Biol 2012;9:850-9.

[8] Yuana Y, Bertina RM, Osanto S. Pre-analytical and analytical issues in the analysis of blood microparticles. Thromb Haemost 2011;105:396-408.

[9] Lacroix R, Judicone C, Poncelet P, Robert S, Arnaud L, Sampol J, et al. Impact of pre-analytical parameters on the measurement of circulating microparticles: towards standardization of protocol. J Thromb Haemost 2012;10:437-46.

[10] Robert S, Poncelet P, Lacroix R, Arnaud L, Giraudo L, Hauchard A, et al. Standardization of platelet-derived microparticle counting using calibrated beads and a Cytomics FC500 routine flow cytometer: a first step towards multicenter studies? J Thromb Haemost 2009; 7:190-7.

[11] Artoni A, Merati G, Padovan L, Scalambrino E, Chantarangkul V, Tripodi A. Residual platelets are the main determinants of microparticles count in frozen-thawed plasma. Thromb Res 2012;130:561-2.

[12] György B, Módos K, Pállinger E, Pálóczi K, Pásztói M, Misják P, et al. Detection and isolation of cell-derived microparticles are compromised by protein complexes due to shared biophysical parameters. Blood 2011;117:e39-48.

[13] Larson MC, Luthi MR, Hogg N, Hillery CA. Calcium-phosphate microprecipitates mimic microparticles when examined with flow cytometry. Cytometry A 2013;83:242-50.

[14] van Ierssel SH, Van Craenenbroeck EM, Conraads VM, Van Tendeloo VF, Vrints CJ, Jorens PG, et al. Flow cytometric detection of endothelial microparticles (EMP): effects of centrifugation and storage alter with the phenotype studied. Thromb Res 2010;125:332-9.

[15] Smalley DM, Root KE, Cho H, Ross MM, Ley K. Proteomic discovery of 21 proteins expressed in human plasma-derived but not platelet-derived microparticles. Thromb Haemost 2007;97:67-80.

[16] Boilard E, Nigrovic PA, Larabee K, Watts GF, Coblyn JS, Weinblatt ME, et al. Platelets amplify inflammation in arthritis via collagen-dependent microparticle production. Science 2010;327:580-3.

[17] Jayachandran M, Miller VM, Heit JA, Owen WG. Methodology for isolation, identification and characterization of microvesicles in peripheral blood. J Immunol Methods 2012;375:207-14.

[18] Emmerechts J, Jacobs L, Van Kerckhoven S, Loyen S, Mathieu C, Fierens F, et al. Air pollution-associated procoagulant changes: the role of circulating microvesicles. J Thromb Haemost 2012;10:96-106.

[19] Connor DE, Exner T, Ma DD, Joseph JE. The majority of circulating platelet-derived microparticles fail to bind annexin $\mathrm{V}$, lack phospholipid-dependent procoagulant activity and demonstrate greater expression of glycoprotein Ib. Thromb Haemost 2010;103:1044-52.

[20] van der Pol E, van Gemert MJ, Sturk A, Nieuwland R, van Leeuwen TG. Single vs. swarm detection of microparticles and exosomes by flow cytometry. J Thromb Haemost 2012;10:919-30.

[21] van der Pol E, Böing AN, Harrison P, Sturk A, Nieuwland R. Classification, functions, and clinical relevance of extracellular vesicles. Pharmacol Rev 2012;64:676-705.

[22] Maurer-Spurej E, Pfeiler G, Maurer N, Lindner H, Glatter O, Devine DV. Room temperature activates human blood platelets. Lab Invest 2001;81: 581-92.

[23] Bretelle F, Sabatier F, Desprez D, Camoin L, Grunebaum L, Combes V, et al. Circulating microparticles: a marker of procoagulant state in normal pregnancy and pregnancy complicated by preeclampsia or intrauterine growth restriction. Thromb Haemost 2003;89:486-92.

[24] Arroyo JD, Chevillet JR, Kroh EM, Ruf IK, Pritchard CC, Gibson DF, et al. Argonaute 2 complexes carry a population of circulating microRNAs independent of vesicles in human plasma. Proc Natl Acad Sci U S A 2011; 108:5003-8.

[25] Redova M, Poprach A, Nekvindova J, Iliev R, Radova L, Lakomy R, et al. Circulating miR-378 and miR-451 in serum are potential biomarkers for renal cell carcinoma. J Transl Med 2012;10:55. 
[26] Henriksson CE, Klingenberg O, Hellum M, Landsverk KS, Joø GB, Westvik AB, et al. Calcium ionophore-induced de-encryption of tissue factor in monocytes is associated with extensive cell death. Thromb Res 2007;119:621-30.

[27] Crespin M, Vidal C, Picard F, Lacombe C, Fontenay M. Activation of PAK1/2 during the shedding of platelet microvesicles. Blood Coagul Fibrinolysis 2009; 20:63-70.

[28] Rogers AB, Des Prez RM. The effect of $\mathrm{pH}$ on human platelet aggregation induced by epinephrine and ADP. Proc Soc Exp Biol Med 1972;139:1100-3.
[29] Nachmias VT, Yoshida K, Glennon MC. Lowering pH in blood platelets dissociates myosin phosphorylation from shape change and myosin association with the cytoskeleton. J Cell Biol 1987;105:1761-9.

[30] Marumo M, Suehiro A, Kakishita E, Groschner K, Wakabayashi I. Extracellular pH affects platelet aggregation associated with modulation of store-operated $\mathrm{Ca}(2+)$ entry. Thromb Res 2001;104:353-60.

[31] Owens III AP, Mackman N. MP's and VTE's: Fact or fiction. Thromb Res 2011; 128:505-6. 